\title{
Response to Commentaries on The Art of Communication Through Drawing: The Case of "Mr. R," a Young Man Professing Misanthropy While Longing for Connection With Others
}

\section{On Reading Dr. Kumano’s and Dr. McLeod's Commentaries}

\author{
KAYOKO MURASE ${ }^{\mathrm{a}, \mathrm{b}}$ \\ ${ }^{\mathrm{a}}$ Taishō University \\ b Correspondence regarding this article should be addressed to Kayoko Murase, Nishisugamo 3-20-1, Toshima-ku, \\ Tokyo, 170-8470, Japan. \\ Email: kayoko.murase.phd@gmail.com
}

\begin{abstract}
I would like to express my gratitude for the opportunity to read commentaries by non-Japanese and Japanese commentators and reflect back on both this case and on the basic principles of psychotherapy. Both commentators (McLeod, 2015 and Kumano, 2015) picked up profound themes such as therapist's way of being, the relationship with the client, understanding the client and his experiential world, the use of drawing as a technique, and the succession of cultural tradition of psychotherapy, offering many valuable suggestions through this dialogue on the case of Mr. R. I responded to Dr. Kumano on his questions about how I assessed data from numerous data sources and on what basis I decided to introduce the drawing method. In response to Dr. McLeod's questions, I touched on presence as the therapist's basic stance, therapeutic goals, supervision, mutual portrait drawing, and assessment of client attachment. Finally, I concluded my response by laying out the basic principles of psychotherapy.
\end{abstract}

Key words: drawing; integrative therapy; Japanese client; therapeutic communication; therapeutic relationship; personality disorder

\section{ON READING DR. KUMANO’S COMMENTARY}

\section{Introduction}

Psychotherapy employs psychological theories and techniques with the goal of helping people live even just a little bit more comfortably. Therefore, the goal of psychotherapy remains the same even if theories and techniques as well as the characteristics of the processes that follow from them differ. In the first place, in the practice of psychotherapy, therapists should not assume a certain posture derived a priori from a theoretical stance that emphasizes its theory and technical features before meeting their clients. The cardinal principle of psychotherapy is to apply theories and techniques that match the results of client assessment: The nature of the issues for which the client seeks solution, the factors contributing to the issues, various contextual and background factors, the way the client has lived their life so far, the way in which they perceive their world, their needs and desires, and what resources exist both within and without that can be 
useful to the client's healing and improvement.

Dr. Kumano is a behavioral therapist, yet is very well-versed in a variety of approaches such as psychoanalysis, Naikan therapy, and Morita therapy. Although our approaches are different, I requested his commentary hoping that essential therapeutic factors for psychological helping would be distilled there because of the depth of his understanding. Without failing to meet my expectation, he made numerous valuable suggestions for which I am grateful. In the following, I would like to write my impression along the flow of his commentary.

\section{Method and Technique of Case Study: In the Beginning Was Fact}

At the end of the first session, I came to two conclusions based on evaluating the direction that the client wished to take and what I understood to be the whole picture of the client, as well as data such as treatment and family history. Rather than a synthesis based on treatment history, assessment interviews and testing results, these were more like disparate and scattered bits of information that required my imagination to stitch together to gain an overall perspective. First, I was concerned that psychological interviewing mainly using verbal interaction might result in a superficial debate lodged in the dimension of abstract ideology. Second, R had developed his apparently intelligent and logical linguistic capacity during his prolonged truancy and without much interaction with others; therefore, his words might not truly reflect his own feelings and thoughts accurately, even if they appeared to be based on complex valid reasoning.

For this reason, I came to be of the opinion that drawing might enable him to express his inner world at the pre-conceptual level. Each word can communicate a common meaning that can be shared with others. However, when the verbalization is made it is received as expressing a particular feeling and/or thought of the speaker; therefore, he or she cannot retreat from the utterance or undo it completely. On the other hand, compared to more explicit verbal expression, feelings and thoughts put into the form of drawings are only implicitly expressed; how viewers perceive and receive these through the drawings weighs more.

It is therapeutically useful for the therapist to grasp this implicit meaning skillfully according to the given situation. It is important that at times the therapist can read into the subtle nuances of the client experience only when he or she intentionally refrains from verbalizing what is only implicitly expressed.

For example, soon after the start of therapy, Mr. R drew the portrait of Stalin and the collapsed tulip from which we could infer the image of his father and his mother, and also the family relationship. This seems to have protected Mr. R from the potential inner conflict arising from regret-like feelings about allowing others to know certain background information that he would rather keep to himself, as well as from the desire to be understood by others. Drawing was appropriate for this case because it was expected to reduce the subtle conflict that might have occurred in verbal interactions and it also helped me infer the parent-child relationship. 
I follow a basic stance of employing a relevant method only after grasping the task at hand well, and from there I always try to increase my repertoire of therapeutic techniques. There are times when I develop techniques that are appropriate for particular therapeutic situations by examining the benefits and drawbacks. Dinner with Mr. R's family, in this case, was one such example. I had anticipated the potential problems of losing an adequate psychological distance with him and also the risk of increased dependence resulting from over-stimulating excessive expectation. However, I determined that the relational foundation wouldn't break down as long as I firmly maintained a neutral stance to oversee the whole situation and at the same time to imaginatively envision and understand his experiential world.

Mr. R who protected himself awkwardly by holding on to an excessively conceptual stance recounted, "Now for the first time in my life I realize the meaning of words like "family circle" and "evening meal" (p. 100). This tells us that therapy generated an experience in which words and their meanings were felt and understood by him. Mr. R's family had an experience of sitting at a table together, chatting as a family, and sharing their thoughts and feelings for the first time in several years. They were starting to experience the continuity of time connecting present to future instead of simply getting by each moment.

This was a somewhat bold attempt, yet it turned out be an effective one. I did not provide direct guidance or suggestion to Mr. R or his family. I only did this indirectly by arranging its setting. Through this experience, they became aware of the seed of resilience within themselves and their willingness to take on their issues on their own. Dr. Kumano stated that there are more similarities than differences between this case and those typical of behavioral therapy, even though the form is different; my cardinal principle of psychotherapy, in the beginning is fact, was confirmed and shared by Dr. Kumano.

\section{Facilitative Conditions for Change: Therapist Development and Generativity}

Dr. Kumano pointed out two facilitative conditions in this case: one is use of the drawing technique suited to this client, and the other is the unwavering genuineness of the therapist. I would like to discuss this further from a more general perspective. The role of the therapist is to facilitate client change process by continuously tracking and assessing the client and applying techniques relevant and congruous to a particular situation. At the same time, the therapist values the client's sense of agency, receives what the client expresses and communicates, and supports the client in becoming aware of their substantiated self-esteem and self-efficacy as the therapy proceeds.

I also found deeply intriguing the fact that in order to discuss an essential theme Dr. Kumano used the expression, "passing on cultural tradition" instead of "professional development of the therapist" or "therapist training". He used this expression to refer to two things. One concerned my personal view of therapy that a therapist needs to make incessant efforts at professional development and that in spite of that, their development starts and ends with them. The other is that Mr. R's nephew, who learned from his mother of his uncle's dramatic transformation and the vividly experienced impact of psychotherapy, came himself to desire to become part of the psychological helping professions. It reminded me of the many 
potential ways that psychotherapy can have an impact. The possibility exists that the positive impact can be implicitly and faintly communicated not only to the client, but also to more than one other individual.

In order to provide evidence and to be accountable for my therapeutic action, I report facts that are not amenable for quantification in a way that facts speak for themselves; in other words, I try to maintain the perspective of viewing my actions as objectively as possible, while at the same time achieving a stance of aligning myself with the client and to see, feel, and think of the world together. Furthermore, in case studies, I seek to use expressions that describe both the idiosyncratic nature of such facts and also to uncover universality within them.

\section{ON READING DR. MCLEOD’S COMMENTARY}

I was deeply touched by the commentary of Dr. McLeod for referring to the importance of presence as a total way of being for the therapist in psychotherapy, and for pointing out that it was an undercurrent for this case study. Most of the discussions on therapist presence in psychotherapy have focused on it as a component of relationship or as an aspect of technique, and to a much lesser extent as a basic relational attitude of a person. Perhaps it is because this is too obvious, and the understanding of it might still remain at the level of what is commonly referred as the "character" of a person.

I have on many occasions observed that no matter what the particular brand of therapy concerned, its effectiveness varies greatly depending on the therapist as a person. Therefore, I have always wanted to explore a therapist's ways of being and the characteristics that impact the process of therapy and facilitate client change without foreclosing the exploration of its nature by capturing it with a general overarching common term such as “character”. Dr. McLeod also pointed out, with much sensitivity and meticulousness, techniques used in this case and their potential by perusing much relevant literature in the field.

I would like to briefly answer questions that Dr. McLeod raised at the end of his commentary and then would like to comment on my opinion about my action (e.g., inviting Mr. $\mathrm{R}$ for a meal) that apparently fails to meet basic principles of psychotherapy and yet that brought about the opportunity for transformation rather than problems of so-called client acting out.

\section{Responses to the Questions}

\section{How and When Was the Goal of Therapy Discussed?}

According to his father, Mr. R had not been able to maintain a stable therapeutic relationship at many clinical and consultation facilities and there was no sign of improvement. He commanded me to bring any change from this state, which I felt that I had no choice but to accept.

Mr. R. said that he had "no trust in people" (p. 90) and that he wanted to try talking with "Doctor Murase's lady" (p. 87). I told him in the beginning of the first session that we 
would "start off with what he wants to talk about without pushing too much until we find a turning point.” (Mr. R nodded with a somewhat skeptical expression on his face. I sensed the air of him testing me. Therefore, I thought to myself that I needed to be honest and authentic without being threatening to Mr. R. or bruising his confidence.)

\section{In working on Such a Difficult Case, Was Supervision Necessary in Your Opinion?}

I was supervised by Takao Murase (my late husband, who passed away in 1998) who was a supervisor for the therapist with whom Mr. R. had worked right before me and also by Takeo Doi, a psychiatrist. (While an assistant professor, Takao Murase founded an outpatient clinic at Tokyo University, which was part of the progressive movements at the time. Soon after, he became dissatisfied with the clinical practice at the clinic and became one of the first staff psychologists at a psychiatric hospital seeking research and practice at more challenging settings. He then studied in the United States as a Fullbright scholar and became acquainted with Eugene Gendlin's work. He always ventured out into unexplored territories. After midway into the treatment, I presented this case at a regular meeting of the psychotherapy study group led by Dr. Takeo Doi. The two guides did not give any specific suggestions or opinions. However, their presence was in and of itself a support to me.

\section{Why Did I Think Drawing Was Appropriate for This Case? What is the "Mutual Portrait}

\section{Drawing Method”? Any References in English?}

Mr. R. was highly intelligent; however, he had spent most of his developing years in a withdrawn state. I observed that his seemingly intelligent and sharp verbal expression had no firm grounding, but was instead lodged in the superficial ideological dimension. I was afraid that we might end up engaged in an ideological debate, trading barbs with each other. I thought of drawing as a method for him to get in touch with his own feelings and particularly those inner feelings and thoughts that are hard to verbalize, for him to accept himself and to gain a sense of coherence. I thought drawing could help him express those inner feelings and thought contents that cannot be easily verbalized. In addition, since drawing does not have to rely on words, both Mr. R. and the therapist could imagine and grasp what can only be vaguely and faintly received, thereby invoking less resistance as we went along.

There is no reference in English that I know of on the mutual portrait drawing method. There is one book in Japanese, which I wrote (Murase, 2005).

I received a request to design and provide psychological intervention from a clinical inpatient facility for the hearing impaired with co-morbid disorders. Many of the patients had multiple disorders, including developmental disorders, mental disorders, and physical disorders. Schooling was also considerably difficult: they didn't use sign language, writing, or fingerwriting, so communication was not easy and they experienced severe difficulties in living. They lived their days in vain and the staff at the facility were demoralized. I was asked to help these patients regain vitality and enjoy their lives in spite of the many hardships.

I was at a loss not knowing what I could do in the face of those inpatients who had no 
method of communication, had no meaning in their life, and passed each day with little sense of vitality. I gave each person a gift, which was a hand-mirror so that they could be aware of themselves, and I invited them to draw their self-portraits. This attempt awakened consciousness of their self and of me. Though somewhat perplexed initially, they began to engage in grooming behaviors such as shaving, fixing their hair, and straightening themselves up when meeting others. An awareness of other people and the inclination to take care of themselves started to emerge. In the midst of these changes, I was further asked to provide one-shot, 30-minute interventions for those patients who exhibited severe behavioral problems such as stubborn withdrawal, intense fear of others resulting in uncontrollable crying, and impulsive aggression toward others.

It seemed like an impossible request to me. But seeing people who sobbed, screamed to fend off others, or sat catatonically like statues all day long except at meal and bath time, I also really wished I could help them feel even a tiny joy in living. That is when I came up with a variation of the drawing method, in which the patients and I would draw each other's portraits and exchange them in turn. Instead of simply portraying what I actually saw, I experimented with drawing what the person would look like when they felt good, or when they smiled.

The patients received this much better than I had imagined. The patients, who initially drew my face mechanically or sloppily with Japanese characters which looked like a face, redid their drawings to make the face look more real after seeing the portraits that I drew of them in which they were smiling. One person came close to me holding crayons up to my clothes to recreate the same pale blue color. They all put my drawings of them up on the wall with great care.

One family told me tearfully, "You drew my son more pleasant and more like an adult man." Following this, many patients became more aware of things around them, and the climate in which they mutually cared for one another and helped those who had more severe impairments started to grow little by little. Among the exhausted and dispirited staff, opinions such as "people change when we approach them differently," and attempts to change their approach to their patients started to emerge.

\section{In the First Phone Call, How Did I Assess Mr. R’s Attachment?}

I felt that Mr. R fervently wished to be treated as a person in the same world. Whether his previous therapists intended or not, he sensed manipulation in their utterances that they, aware of therapeutic goals as they tweeked their responses, could not avoid betraying. Mr. R. was in a painful self-contradictory state in which he sincerely craved for genuine communication with others while also being unable to accept his role as a client when the only interpersonal contact he had was with his therapists.

\section{THE PRINCIPLES OF THERAPEUTIC CHANGE}

Due to the limitation of space, instead of answering the other very valuable suggestions and questions raised by Dr. McLeod beside those four questions above, I would like to conclude 
my response by summarizing the principles of therapeutic change that form the foundation of my clinical work.

I value keeping the time and place dimensions of therapeutic context in perspective at all times. First, what kind of historical time is the present? At what stage of the life cycle is the client? At what stage of professional development and what stage of life cycle is the therapist? What expertise has she or he achieved? The therapist needs to understand these issues in perspective. Furthermore, therapists need to be aware and recognize in what kind of time flow psychotherapy with a particular client is unfolding, what state it is in, and what factors are impacting its time flow.

Second, what place or clinical setting (organization) does the therapist work in and what are the functions and rules of such a clinical setting? The therapist needs to fully recognize the characteristics of the clinical setting. Third, the therapist needs to understand his or her position. What position or role does the therapist play in the organization? The therapist needs to be cognizant of what he or she can do and cannot do, what she or he must not do, to what extent he or she can be responsible, and how she or he gets consultation and guidance. It is desirable to acquire capacity to make quick judgments and be mindful of the above three things.

Next, it is important to engage in continuous assessment. The therapist is continuously assessing the whole situation that the client is in, those significant others who have meaningful relationships with the client, and the total environment of the client, while valuing the client's agency. This involves the high quality receptivity (or passivity) of the therapist. The therapist attempts to keep tracking the moment-to-moment changing process and grasp the nature of emerging change. While seeing the whole situation in perspective, the therapist still focuses on what is important in that particular moment. In working with clients, therapists are expected to simultaneously maintain three person-perspectives in balance.

The first person-perspective involves aligning the therapist's experience with that of the client by imaginatively entering the client's experiential world, seeing the world from the client's perspective, sharing the view, and understanding the client's feelings through a so-called visceral sense.

The second person-perspective is one that involves relationality with some sense of distance rather than oneness, which might be expressed when the therapist says to the client: "You are experiencing such difficulty, suffering, sadness, or pain in your life."

The third-person perspective involves the therapist's attempts to capture and understand the client's state by objectifying it. It is a starting point from which the whole picture of the client, the features of the environment, the origin of the client's problems are indicated using the expression in common, familiar language.

Clinical work of excellence requires maintaining these three person-perspectives in balance, in my view. I consider it essential to have these three perspectives and to be accurately aware of the content of one's feelings and thoughts that emerge from within for enabling clinical 
judgment to be precise. So I have practiced it. When you gain a sense of basic balance, you can make accurate judgments, such as how much territory can the therapist responsibly cover before either stepping back and referring the client to relevant professionals and/or starting to work in liaison with others. I did not invite Mr. R to my house for dinner just because the idea popped into my head. What did I need to note in executing this plan? I exhaustively examined both advantages and disadvantages of executing this plan. As a result, I decided to carry it out despite many cautions because the potential benefits of this attempt outweighed the concerns.

The fundamentals of psychotherapy that are needed are: to hold in mind the above three person-perspectives at the same time and to bring them into operation; to be empathic while not losing one's calm ability to think rationally; to encounter the client as a person with difficulty in living; and overall, to know the awe of touching someone's heart and wondering how one can improve his or her own shortcomings.

\section{REFERENCES}

Kumano, H. (2015). Discontinuous change facilitated by emotional expression through drawing and the accurate verbal responsiveness of the therapist. Pragmatic Case Studies in Psychotherapy, 11(4), Article 3, 255-263. Available: http://pcsp.libraries.rutgers.edu

Murase, K. (2005). Chokakushogaisha eno Togoteki Apurochi: Komyunikeshon no Itoguchi wo Motomete. [Integrative psychotherapy to those with auditory challenges: Searching for the window of communication]. Tokyo, Japan: Nihon Hyoron Corp.

McLeod, J. (2015). Narrative case studies and practice-based learning: Reflections on the case of "Mr. R.” Pragmatic Case Studies in Psychotherapy, 11 (4), Article 2, 239-254. Available: http://pcsp.libraries.rutgers.edu 